\title{
OBTAINING U.S. COPYRIGHT REGISTRATION FOR THE Elara Square Cut-Cornered BRILLIANT DIAMOND
}

\author{
Howard B. Rockman
}

In 2000, the U.S. Copyright Office granted what is believed to be the first copyright registration covering a gemstone design. Previously, the Copyright Office maintained that cut designs lacked artistic or sculptural authorship, and that gemstone faceting was a non-copyrightable array of common geometrical shapes in a three-dimensional object. The application to obtain copyright registration for the Elara-a square cut-cornered brilliant diamond design-was initially rejected on similar grounds. However, the position of the copyright examiner was successfully appealed to the highest level of the Copyright Office, which ultimately decided that copyright registration for the Elara cut was appropriate. This experience shows that there may be a new intellectual property vehicle available for manufacturers to protect proprietary cut designs.

A recent Gems ↔) Gemology article (Overton, 2002) stated that U.S. federal courts require more original artistic expression to establish a copyright than exists in diamond cut designs. While court decisions may presently indicate that gemstone designs are not copyrightable, the U.S. Copyright Office recently issued copyright registration No. VA-1-104-147 (effective June 29, 2000) for the Elara square cut-cornered brilliant diamond cut (figure 1), produced and marketed by Kuwayama Europe of Antwerp, Belgium, and its U.S. affiliate, Elara Diamond U.S.A. According to the U.S. Register of Copyrights, the Elara diamond cut is the first gemstone design the Copyright Office has ever registered (M. Peters, pers. comm., Nov. 6, 2001). This article summarizes the background and history of the Elara copyright registration process.

\section{THE COPYRIGHT REGISTRATION PROCESS}

U.S. copyright law protects works of "authorship" such as books and movies, as well as sculptures, computer programs, photographs, and myriad additional media that continually develop as art and technology advance (see U.S. Code, Title 17; information in this article is drawn from that source). The copyright holder has the exclusive right to control reproduction of the expression embodied in his or her artistic creation after it has been fixed in a tangible medium such as the print in a book, the images on a reel of film, or the electronic pattern on a computer disc, CD, or DVD. This includes control over copying of the work, the preparation of derivative works, distribution of copies to the public, public performance, and public display. Only the copyright holder can sell or license these rights to other persons or entities. The term of a copyright begins at the moment of fixation in a "tangible medium of expression" and currently runs for the life of the author plus 70 years. In the case of "works for hire" (those created by employees for their employers or by outside authors working under a written contract), it runs for a total of 95 years.

When considering a work comprised of several elements, such as the facets of a gemstone design, the existence of copyright rests on the originality inherent in the coordination or arrangement of those specific elements that, in total, comprise the new design. While individual components may not be protected

See end of article for About the Author and Acknowledgments. Gems \& Gemology, Vol. 39, No. 3, pp. 210-213.

(C) 2003 Gemological Institute of America 


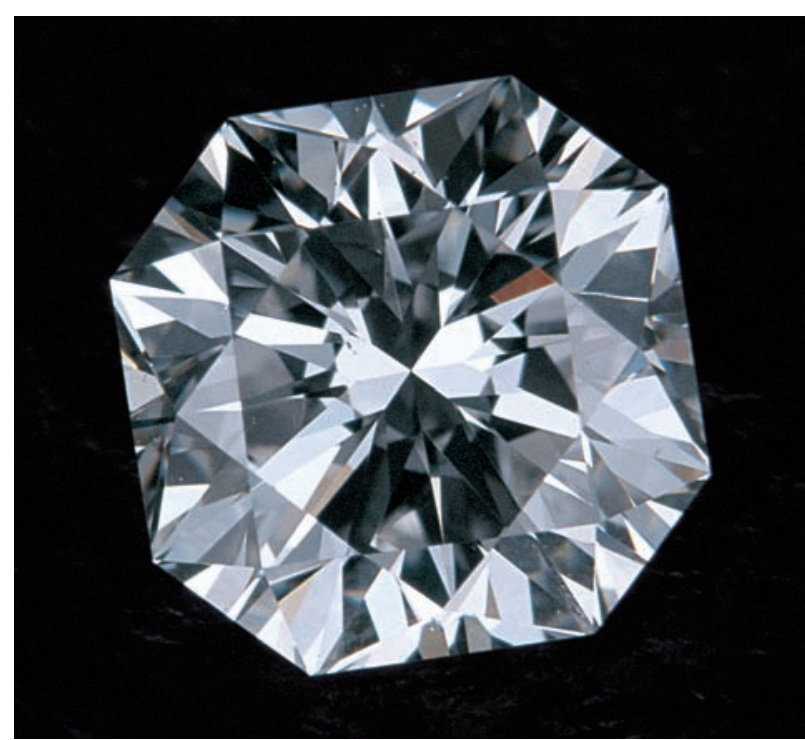

Figure 1. The Elara diamond is the first diamond cut design to be awarded a U.S. copyright registration. Courtesy of Elara Diamond Corp.

by copyright, such protection may be triggered by the particular way in which the underlying elements combine to arrive at the finished design. It is the originality of arrangement that determines whether or not it is eligible for copyright protection. In other words, while the squares, triangles, and parallelograms that make up a gemstone facet design may not be individually protectable by copyright, an original and innovative arrangement of them may be protectable.

At any time during the term of a copyright, the copyright owner may seek federal registration of his or her claim by applying to the U.S. Copyright Office, paying the designated fee, and depositing a specimen of the work with the application. A copyright examiner will review the application to ensure that it covers a work that is indeed copyrightable subject matter under the law. If all requirements are correctly set forth in the application, and the submitted specimen establishes that the work constitutes copyrightable subject matter, a Certificate of Registration is issued. If the examiner makes a negative finding on any of these issues, registration is refused. This refusal may be appealed to a first level and, if necessary, to a second, higher level within the Copyright Office.

One can hold and own (and transfer and license) a copyright without obtaining registration for it, but a copyright owner must obtain federal registration before taking action to enforce the copyright against an infringer. However, an infringement action can be brought if a copyright registration application has been filed but refused, as long as a copy of the infringement complaint is furnished to the Copy-

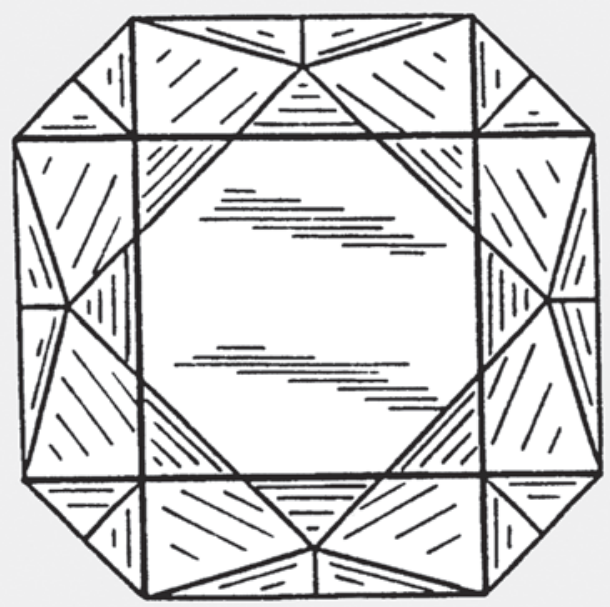

Figure 2. This facet diagram shows the top view of the design of the Elara cut as registered with the U.S. Copyright Office.

right Office. In addition, the timing of the registration application after first publication affects the type of damages that may be recovered. In most foreign countries, there is no comparable registration system; thus, no prior registration is required to enforce a copyright outside the U.S.

\section{FIRST APPLICATION FOR REGISTRATION OF THE ELARA DIAMOND AND FIRST APPEAL}

The copyright registration procedure for the Elara diamond began on June 29, 2000, when an application (with photos) was filed on behalf of Kuwayama Europe, covering a gemstone design (see figure 2) then known as the Flanders Brilliant /since changed to Elara).

The Copyright Office initially rejected the application (J. H. Ashley, pers. comm., Nov. 13, 2000) and repeated its refusal to register following a request for reconsideration (the first appeal; V. Giroux, pers. comm., May 25, 2001). It stated that the diamond design lacked the artistic or sculptural authorship necessary to support a copyright registration, and based its conclusions on several factors.

First, the examiner believed that the subject of registration was the process of faceting the gem, and ideas, concepts, and processes that may be embodied in a work are not subject to copyright protection (see U.S. Code title 17, section 102[b]).

Second, to meet the originality requirement for copyright registration, a work must possess more than a de minimis level of creativity. The examiner 
held that, as discussed above, copyright law does not protect familiar geometric shapes or patterns, nor minor variations of them (see Code of Federal Regulations, title 37, section 202.1), and that it was normal procedure to refuse registration for gemstones when the faceting may be perceived to be a combination of standard or common geometrical shapes in three-dimensional form.

Third, the Copyright Office analyzed the design for copyrightability in two ways. First, the top, bottom, front, and side views were treated as separate designs. Second, the entire gemstone was considered as a whole entity. The examiner found insufficient originality in either of these analyses, stating that the overall arrangement of the facets, taken individually or as a unified whole, did not constitute a copyrightable work.

Fourth, the Copyright Office stated that while a work may be unique and distinctive, while considerable time, effort, and expense may have gone into its creation, or while it may be commercially valuable or successful, these facts do not in themselves establish original artistic authorship (V. Giroux, pers. comm., May 25, 2001).

\section{THE SECOND APPEAL}

Prior to receiving the action and comments of the copyright examiner, the creator of the design, Johan d'Haene of Antwerp, had explained to the author the precise steps he took to create the Elara cut. His ultimate aim was to incorporate into a square cut-cornered diamond shape as many of the optical qualities of the round brilliant as possible. Based on this information, the author judged that sufficient creativity and authorship existed in the design to support a second appeal in an effort to reverse the decision of the copyright examiner. On August 1, 2001, the author submitted a response and request for reconsideration of the refusal to register the copyright claim in this design.

The appeal was based on two grounds. First, it maintained that the Elara cut possessed sufficient creative authorship in the origination of its design. Second, it confirmed that registration was not being sought to cover the process by which the facets were applied to the rough diamond. Instead, the author argued that the steps taken in extrapolating the standard round brilliant to the Elara design constituted acts of sculptural authorship.

The appeal began by describing the basics of the round brilliant cut, as described in Tolkowsky (1919), for maximizing brilliance and fire. As a foun- dation for the creativity and authorship behind the Elara design, the author's brief set forth Mr. d'Haene's extensive experience with De Beers and the Diamond High Council (HRD) in Antwerp. During the 1980s, while a partner of a Belgian company engaged in selecting and buying diamonds for the Japanese market, he saw the need to design new diamond cuts for Japanese tastes, one of which involved the concept of "squaring the circle."

In creating this design, certain crown facet lines were extended outside of the original circle and connected to form a new, square outline with cut corners, the pattern repeating itself for each quadrant of the crown. The final cut evolved from trial and error through several different designs. The results of Mr. $d^{\prime}$ Haene's design efforts are shown in figure 2.

\section{ARGUING THE APPEAL}

In pressing the appeal, we argued that each step in the creation of the Elara cut was the result of artistic design efforts, including personal independent creative thoughts that led to certain facet arrangements. We established that design, in this particular case, went far beyond the level of creativity required to support copyright registration. We also specifically pointed out that the final design was motivated in substantial part by an attempt to create an original work that would convey a visual impression that had not been seen before. Moreover, it would present a markedly different visual image compared to other diamond designs on the market at that time. We also argued that the design retained a significant degree of the brilliance that can be produced by the round brilliant cut.

The description of the specific design steps also supported our contention that the design was not based on a familiar geometric shape, but a combination of facet shapes in a specific, complex arrangement providing the finished design with its unique character. We argued that the creative expression embodied in the design was capable of standing alone as an independent copyrightable work, and not an agglomeration of several standard forms or shapes with minor linear or spatial variations. We specifically pointed out to the Copyright Office that the design comprised originality of facet coordination and arrangement, whereby the shape and position of each facet in the total design coordinated with other facet shapes and positions to provide an eye-catching attractive design.

The requisite authorship for copyright registration was shown to be the particular steps taken in 
the extrapolation of the round brilliant to the square brilliant design, as a result of the effort and thought of Mr. d'Haene. We argued that the totality of the subject diamond design, considered as a whole, produces an aesthetic effect and appearance that conveys to the viewer the beauty of design.

\section{THE DECISION}

On November 6, 2001 (M. Peters, pers. comm.), the Register of Copyrights responded to our appeal as follows:

\begin{abstract}
After careful consideration, the Copyright Office Board of Appeals has decided to register the Flanders Brilliant (ELARA) diamond. The registration is only for the three-dimensional faceted stone because of the complexity of the overall sculptural configuration. We rarely see such complexity in gemstones used in jewelry design, and we cannot recall previously making such a registration. The registration of course does not cover any aspect of the faceting process.
\end{abstract}

In its letter, the Copyright Office also noted that courts have held that copyright protection in this type of work may be limited, particularly where the copyrightable work comprises a unique combination and arrangement of otherwise un-copyrightable elements in the particular design. This means that copyright protection only extends to the design as a whole; others may freely use the individual, un-copyrightable, elements to come up with a new gemstone design having a different overall design and appearance.

\section{CONCLUSION}

It remains to be seen whether the Copyright Office's determination that certain gemstone designs embodying acts of creative authorship are copyrightable sub- ject matter will be followed by the U.S. federal courts, which have the inherent power to overturn such decisions. And since this is the first gem design to be registered, it is also unclear where the courts will draw the line on infringement, that is, how close competitors may come to a copyrighted gem design without infringing on the copyright. Another unanswered issue is the degree to which published, and thus copyrighted, collections of faceting diagrams for hobbyists provide protection for the designs therein.

Gemstone designers hoping to apply for copyright registration for a new design should record the mental, geometric, artistic, and analytical processes leading to the final design in order to support a conclusion of creative and artistic authorship. It would probably be helpful to retain all preliminary drafts of sketches, drawings, and calculations prepared during the design process, should it be necessary to respond to a denial of registration based on a lack of creative authorship. An applicant must also be able to describe how his or her new design differs from existing gemstone designs.

The copyright registration that was obtained by Kuwayama Europe n.v. and Elara Diamond USA, provides its owners with the ability to prevent others from copying the Elara diamond design in their own gemstone products. (Although the Elara design patent is currently being challenged in a U.S. federal court case [see "Elara diamond design patent invalidated. . . " 2003], this case has no bearing on the validity of the copyright.) Kuwayama believes that it fully understands the limitations of the copyright protection afforded by its registration. Nevertheless, the copyright registration obtained on the Elara cut is a pioneering example of how creative authorship, originality, and creativity can combine to produce a gemstone design that meets the requirements of the U.S. copyright laws to support copyright registration.
ABOUT THE AUTHOR

Mr. Rockman (hrockman@btlaw.com) is an intellectual property attorney with the Chicago law firm of Barnes \& Thornburg. As counsel for Kuwayama Europe n.v. of

Antwerp, Belgium, he was instrumental in obtaining the

U.S. Copyright registration for the Elara gemstone design.

\section{ACKNOWLEDGMENTS}

The author thanks Mr. Johan d'Haene of Antwerp, Belgium, for providing information on the planning and design of the Elara diamond cut and for allowing the author to share this information with the gemological community.

\section{REFERENCES}

Code of Federal Regulations, Title 37 (Patents, Trademarks, and Copyrights). Available online at http://www.uspto.gov/web/ offices/tac/tmlaw2.html.

Elara diamond design patent invalidated by U.S. district court (2003) National Jeweler, Vol. 97, No. 18, Sept. 16, pp. 28, 30.

Overton T.W. (2002) Legal protection for proprietary diamond cuts. Gems ↔) Gemology, Vol. 38, No. 4, pp. 310-325.

Tolkowsky M. (1919) Diamond design: A Study of the Reflection of Refraction of Light in a Diamond. E. \& F.N. Spon, London.

United States Code, Title 17: Copyright, Sections 100-1332. Available online at http://www.copyright.gov/title17/index.html. 